\title{
Design and majorization of SOI high temperature pressure sensor
}

\author{
Zhi.Jun. Yao ${ }^{1, ~ a ~, ~ J u n . Z h a o ~}{ }^{2, b}$ and Zheng.Li $i^{3, c^{*}}$ \\ ${ }^{1}$ National-Provincial Laboratory of Special Function Thin Film Materials, and School of Materials \\ Science and Engineering, Xiangtan University, Hunan 411105, China \\ ${ }^{2}$ Center for Semiconductor Particle and photon Imaging Detector Development and Fabrication, \\ Xiangtan University, Xiangtan 411105, China \\ ayzj791582465@126.com, bjz422670934@163.com, 'czhengli58@gmail.com
}

\section{Keywords: pressure sensor, SOI, high-temperature}

Abstract. In high temperature harsh environments, The real-time dynamic measurements of pressure sensors are unable to carry out due to its own limitation. Thus, SOI material and MEMS processing technology are used to solve these problems. Mechanical properties stability and reliability of the sensor structure with SOI are much improved. The new high temperature pressure sensor proposed in this paper, was been studied by means of theory and simulations. The main problem, the reverse of PN junction, caused by the intrinsic excitation of the semiconductor in high temperature, was solved using the silicon on insulator (SOI) material. Then the stability and reliability of the mechanical structure of the sensitive head is guaranteed with the Micro-electromechanical system (MEMS) fabrication process. Using high temperature metal materials as the metal wire material, the package of the pressure sensor can withstand high temperature in harsh environments. The stability and sensitivity of our pressure sensors are much improved in high temperature harsh environment.

By ueing the designing principles of high temperature pressure sensors, together with theoretics and simulations, optimism designs were worked out for various parts of sensors for harsh environments. Performance parameters and maximum operation temperatures of SOI high temperature pressure sesors studied in this paper were obtained according to test results. This work provides insights for the design of sensors that can stand for even higher temperatures.

\section{Introduction}

Nowadays, pressure sensors are often used in modern industrial production to detect the performance of equipment and products. Among them, the pressure-resistant pressure sensors are used in industrial production, aerospace, etc[1]. However, these pressure sensors can only work under normal temperature and are easily to fail at high temperatures, which would not meet the developing needs for modern industrial, military and aerospace industries[2]. Aim of this paper is need to solve the problem of pressure measurement in high temperature environments.

The main reason that silicon materials are used to fabricate the high temperature pressure sensors can be listed as follows: (1) The monocrystalline silicon resistance and substrate are effectively isolated at high temperature without affecting the main performance of the sensors; (2) Due to its good stability, silicon based pressure sensor can be produced on a large scale and low cost; And there are two advantages of the silicon on insulator (SOI) high temperature pressure sensors, firstly, SOI materials have excellent properties, secondly, The pressure sensors are mainly based on the piezoresistive effect of the material, while the noise of pressure sensors with monocrystalline silicon as raw materials for processing preparation are far less than that with other materials[3]. 
In conclusion. In the new field of high temperature pressure measurement, compared with other materials, the high temperature pressure sensor which made of SOI materials have many advantages, so we use SOI to produce high temperature pressure sensors.

\section{optimizing of SOI high temperature pressure sensor}

Using the a finite element analysis (FEA) simulation tool, we can simulate $\mathrm{C}$ shape pressure sensor structure. Shown in Fig. $1 \mathrm{a}$ and $\mathrm{b}$ are sensitive sensor chip of sectional figure and positive sectional. After the argument, $\mathrm{C}$ shape silicon cup technology is simple and stable. SOI materials are $\mathrm{a} \mathrm{SiO}_{2}$ on a layer of monocrystalline silicon wafer materials, The p-type silicon and n-type substrate was isolated by $\mathrm{SiO}_{2}$ insulation layer to improving the reliability of the circuit[4]. SOI piezoresistive pressure sensor, carved four varistors in the direction of [110] and [110], and finally form a wheatstone bridge, on the p-type layer (100) surface) with $\mathrm{SiO}_{2}$ isolation layer, and by the use of pressure sensitive characteristic of single crystal silicon. The silicon on the back of the chip etched the sensitive diaphragm and made the pressure-resistant sensitive chip. Sensor membrane is changed due to outside pressure, and it impacts the stress on the diaphragm, which changes the output of the bridge, and in which, elastic modulus E=190GPa, Poisson's ratio $v=0.278$, Scale range $\mathrm{P}=2 \mathrm{MPa}$ Membrane width $\mathrm{A}=500 \mu \mathrm{m}$. After calculation, the thickness of the diaphragm can be like $\mathrm{H}=30 \mu \mathrm{m}$. The good pressure resistance and mechanical properties of silicon material make it possible to integrate the sensitive components of pressure-resistant pressure sensors and the conversion circuit, and diagrams of top of the silicon core body shows four piezoresistances R1, R2, R3 and R4 (fig.1(b)) which are implanted below the surface of the silicon through ion implantation and diffusion process were placed in (100) crystal plane of $<110>$ crystal direction. The pressure they put on the membrane transform into a change of their resistance, and then use the change of resistance to output the voltage signal through the wheatstone bridge. The resistance R2 and R4 are elongated under external forces so their own resistances were increased, thus they can sense pressure changes. The pressure on the silicon elastomer is transformed into a stress $\mathrm{T}$ on the elastomer, the stress $\mathrm{T}$ further causes the change of the force sensitive resistance that is produced in the elastomer. Resistance $\Delta \mathrm{R}$ is changed into voltage through the whole bridge circuit and finally output $\Delta \mathrm{V}$, because of the integration of the silicon elastomer and the force sensitive resistance, the above-mentioned processes are actually concurrent.

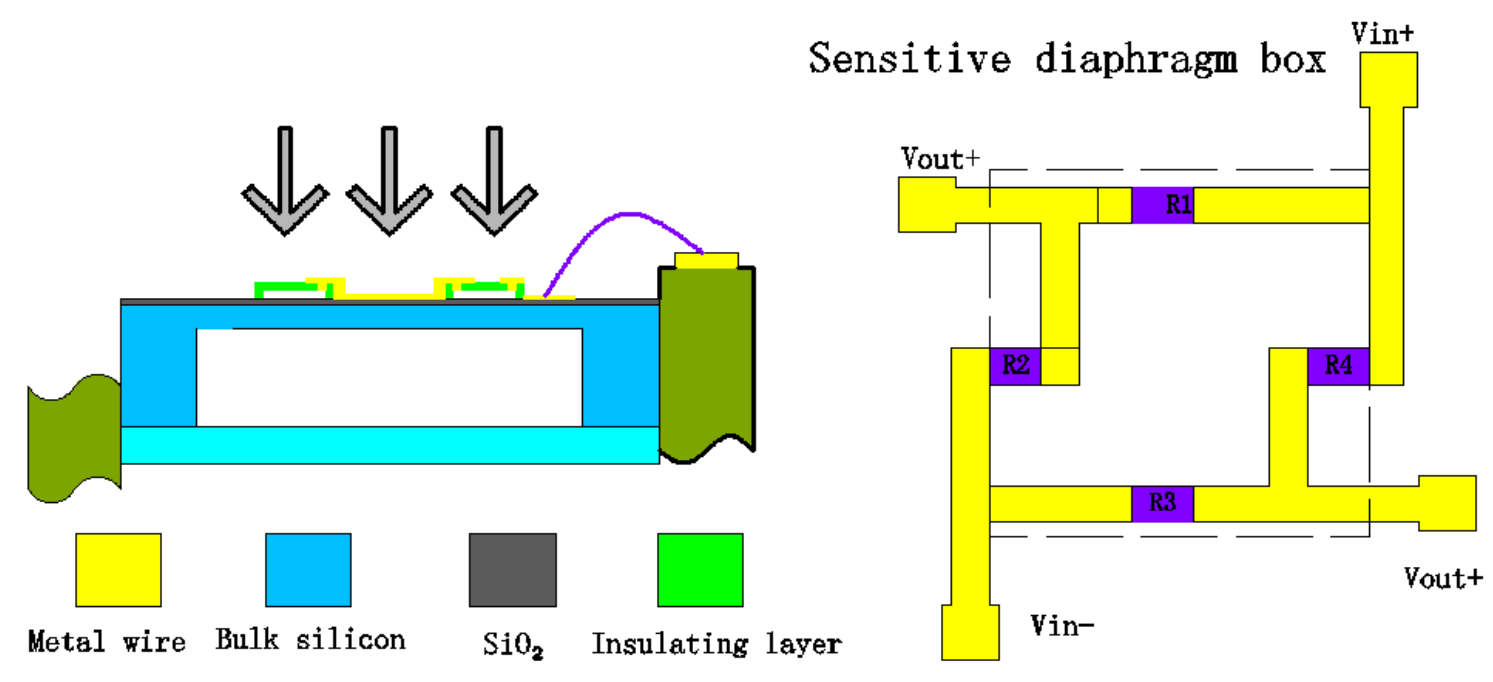

Fig. 1 (a) Sensor chip profile (b) Sensor chip front view 


\section{Stress distribution characteristic}

Shown in figure 2 are the elastic stress in the $\mathrm{x}$ direction and $\mathrm{y}$ direction in $3 \mathrm{D}$ view. The uniformly distributed load is set at $2 \mathrm{Mpa}$ on the upper surface. Fig 2 (a) and (b) are the stress distribution clouds along the $\mathrm{X}$-axis and $\mathrm{Y}$-axis. The elastic stress concentration is on the edge of the membrane.

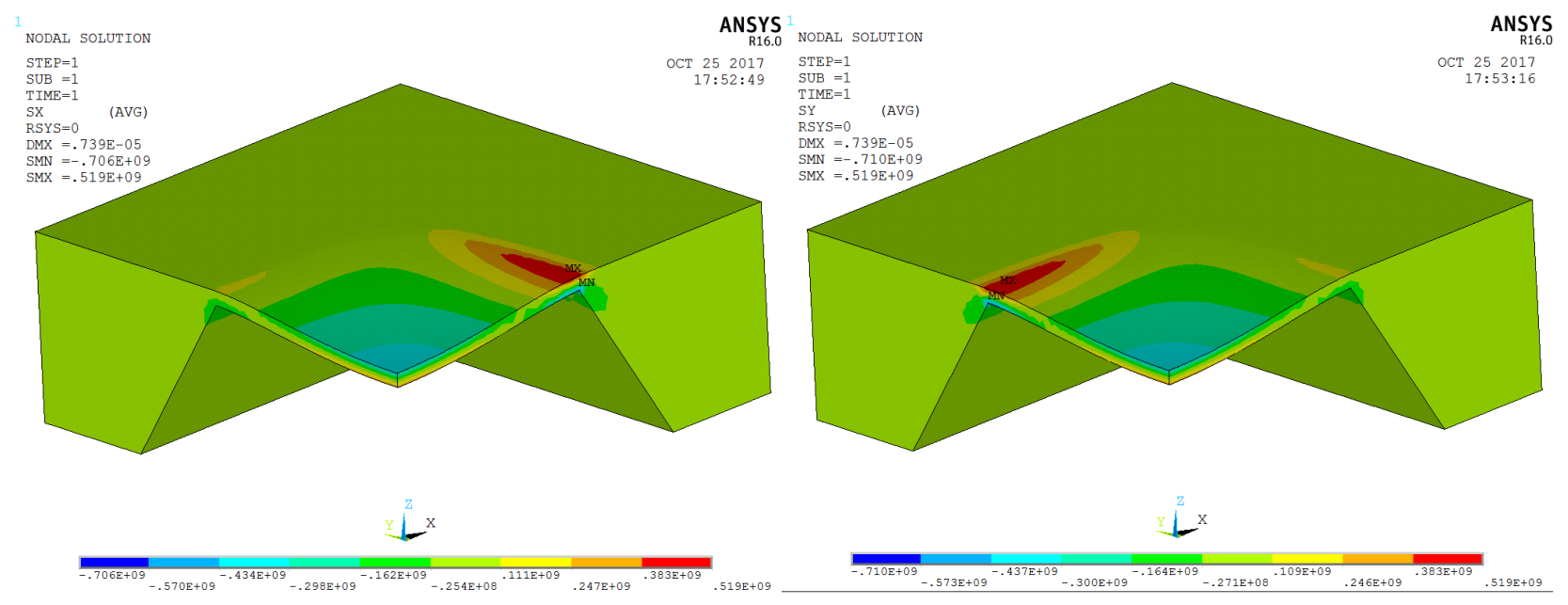

Fig. 2 (a) Stress distribution clouds along the $\mathrm{X}$-axis

(b) The stress distribution clouds along Y-axis

\section{Overload resistance}

The distribution of stress difference on chip surface is very important to study the distribution of compression resistance. Fig.3 (a) shows the results of overload resistance at $2 \mathrm{MPa}$ on the silicon cup surface center, And then it goes down the $\mathrm{X}$-axis, it goes down to a certain value, and then it goes up the $\mathrm{X}$-axis. The same can be said for the change in the differential stress of the Y-axis. Fig.3 (b) shows the absolute value of stress. The stress difference distribution shows that the maximum stress difference is at the center of the four edges of the stress diaphragm, and the maximum value of the stress difference between adjacent sides is the opposite of the equal sign. Therefore, it is only possible to remove the four bridges and other arm resistors in the same direction in the center of the boundary of the four sides of the diaphragm, fig.1 (b) shows that it get the maximum output.

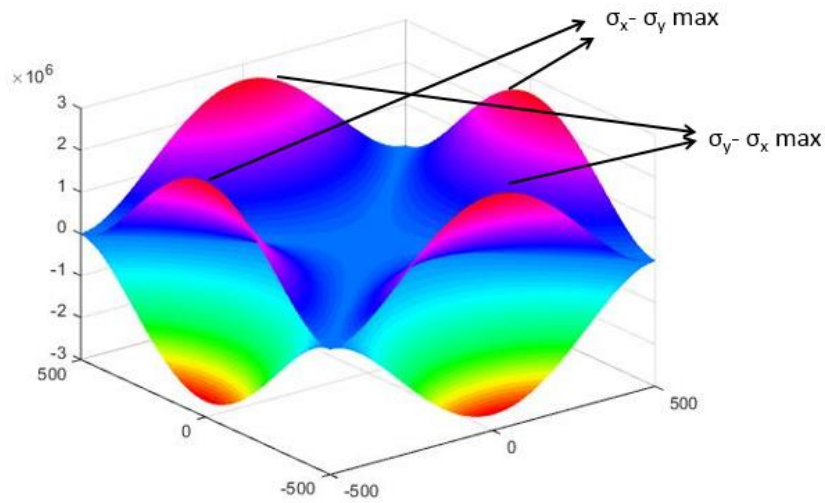

Fig. 3 (a) Max differential stress

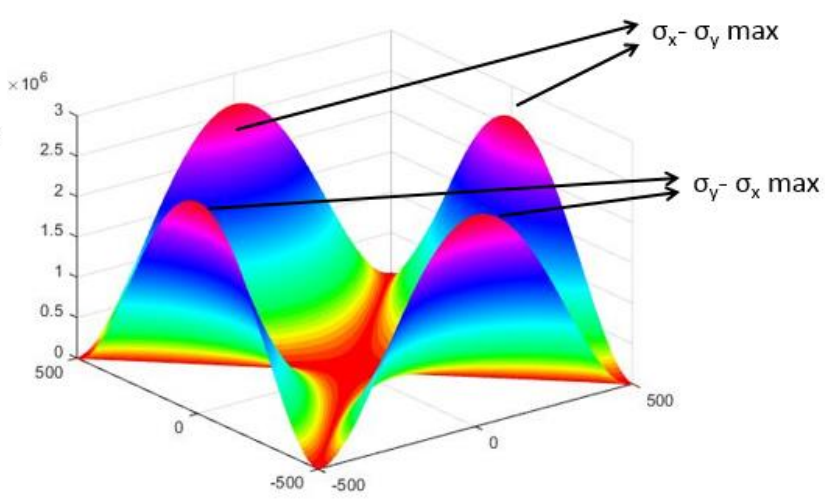

(b) Absolute value differential stress 


\section{Conclusion}

Design and optimization of high temperature pressure sensor have been carried out. This paper combines theoretical analysis with simulations, useing the linear Spares Direct solution of ANSYS. The stability, range, comprehensive precision and instantaneous temperature of optimized SOI high temperature pressure sensor have been improved substantially. The results show that the stress difference of the high temperature pressure sensor is maximum on the surface near the center of the silicon cup. The presure working range is $0 \sim 2 \mathrm{MPa}$, and the overall accuracy is less than 0.1 . At the same time, it was found that the pressure resistance take maximum values on the silicon (100) surface ,and long [110] and [110] directions[5][7], Therefore, these crystal surface and crystal directions are the ideal crystal surface and crystal directions for making sensitive elements, This paper provides a practical basis for future work and developments in high temperature presure sensor.

\section{References}

[1] Weizheng Yuan, Binghe Ma. Micromechanical and microprocessing technology [M]. Xi.An: Northwest industrial university press, 2000, 1-31

[2] Ya Wang, Yi Jia, Qiushui Chen, et al. A Passive Wireless Temperature Sensor for Harsh Environment Applications[J]. Sensors. 8, 2008: 7982-7995.

[3] Ya Suying, Mao Ganru, Qu Hong wei. Nosie effect on characteristics of flip-flop Sensor[J]. Transaction of Tianjin University, 1998, 4(1):82-85

[4] Ke Sun, Yan Lv, Dongxu Zhang. SOI Pressure sensors and applications [J]. Instrumentation technology and sensors, 2009, supplement: 398-399

[5] R.Szilard.Theory and Analysis of Plates: Classical and Numerical Methods Englewood Cliffs [M]. Prentice-Hall.1974, 70-83

[6] L.W.Lin,H.C.Chu,Y.W.Lu. A simulation program for the sensitivity and linearity of piezoresistive pressure sensors [J]. Microelectromemchanical Systems, 1999,8(4) : 514-521

[7] V.Mullem,C.J.Gabriel,K.J.Fujita. Large deflection performallce of surface mieromachined corrugated diaphraphs.International Conference on Solid-State Sensors and Actuators [M]. San Francisco, USA.1991:1014-1017 